\title{
Comparative Study Of Distance Metrics FOR FINDING SKIN COLOR SIMILARITY OF TWO COLOR FACIAL IMAGES
}

\author{
Abul Hasnat ${ }^{1}$, Santanu Halder ${ }^{1}$, D. Bhattacharjee ${ }^{2}$, M. Nasipuri ${ }^{2}$, D. K. \\ $\mathrm{Basu}^{2} *$ \\ ${ }^{1}$ Department of Computer Science and Engineering \\ Government College of Engineering and Textile Technology, Berhampore, \\ India \\ abulhasnat.007@gmail.com.com, sant.halder@gmail.com \\ ${ }^{2}$ Department of Computer Science and Engineering \\ Jadavpur University, Kolkata-700032, India \\ *Former Professor
}

\begin{abstract}
This paper describes the comparative study of performance between the existing distance metrics like Manhattan, Euclidean, Vector Cosine Angle and Modified Euclidean distance for finding the similarity of complexion by calculating the distance between the skin colors of two color facial images. The existing methodologies have been tested on 110 male and 40 female facial images taken from FRAV2D database. To verify the result obtained from the existing methodologies an opinion poll of 100 peoples have been taken. The experimental result shows that the result obtained by the methodologies of Manhattan, Euclidean and Vector Cosine Angle distance contradict the survey result in $80 \%$ cases and for Modified Euclidean distance methodology the contradiction arises in $60 \%$ cases. The present work has been implemented using Matlab 7.
\end{abstract}

\section{KEYWORDS}

Euclidean Distance, Manhattan Distance, Vector Cosine Angle Distance, Distance Metric, Color Histogram, Modified Euclidean Distance.

\section{INTRODUCTION}

Face recognition is a vibrant area of research over the last 20 years and today facial image processing has become an active research area all over the world. A lot of research work on facial feature extraction and face recognition for still and video images using skin color is carried out by Q.H. Thu et al [1], D. Saxe and R. Foulds [2], S. McKenna et al.[3], C. Garcia, G. Tziritas [4], N. Sebe et al.[5], A. Hadid et al.[6 ], D. Bhattacharjee et al. [7], D. Chai, and K.N. Nghan [8] etc. Hence metric is needed sometimes to measure the distance between skin colors of two facial images. In the literature, there exists some distance metrics like Manhattan Distance [9], Euclidean Distance [9] etc to find the distance between two images. In these methodologies less distance signifies more similar images. Vector Cosine Angle Distance (VCAD) [9], Modified Euclidean distance [10] based on color histogram are another approaches to measure the similarity between two images. In Rupak Bhattacharyya et al. (Eds) : ACER 2013, pp. 99-108, 2013. @ CS \& IT-CSCP 2013

DOI : $10.5121 /$ csit.2013.3210 
these two approaches, higher value signifies more similarity between two images. All these methodologies [9][10] are widely used to find a similar image of a given query image by measuring the distance between those images and they give satisfactory results also. But the methodologies are not been tested yet to find the distance between the complexion of two facial images. For this purpose, this paper aims to give a comparison study among the existing distance metrics [9][10] to measure the distance between complexion of two facial images.

This paper is organized as follows: Section II presents the preprocessing required for the facial images in the present work. Section III describes the existing methodologies. Section IV shows the experimental results of comparative study and finally Section V concludes and remarks about some of the aspects analyzed in this paper.

\section{Preprocessing}

As the present work concentrates on the searching an facial color image of similar skin color only, first preprocessing carried out on the face images of FRAV2D database [15] is to crop the facial images from hair to chin (top to bottom) and from right ear to left ear (left to right). This is done to focus on the face skin regions for excluding the background subjects. Fig.1. shows some face images and their cropped images.
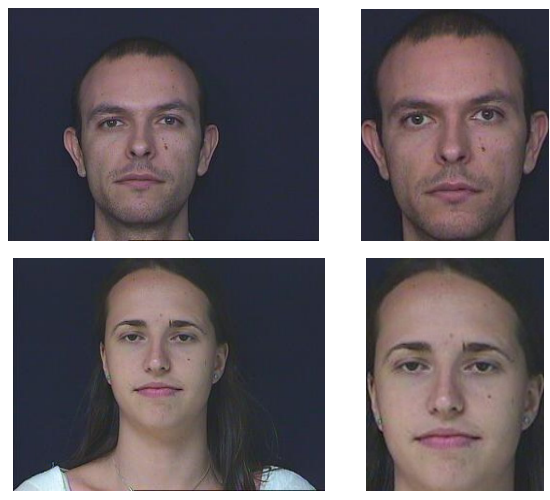

(a)

(b)

Figure 1. Example of preprocessing. (a) Original images (b) Cropped images

As present work concentrates only to express the distance between skin colors of two facial color images, the non skin regions of the facial images are manually removed using Adobe Photoshop CS2 version 9. Fig 2.(b) shows the skin regions of the images in Fig. 2(a). 

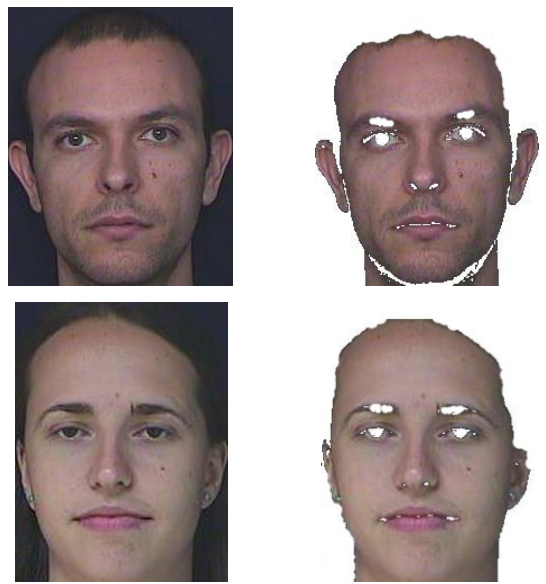

(a)

(b)

Figure 2. Example of facial images and their corresponding skin region (a) Facial images (b)Skin region of facial images

\section{EXISTING METRICS}

There are many different distance metrics to measure similarity/dissimilarity between two images of same size i.e. Manhattan Distance, Euclidean Distance, Vector Cosine Angle Distance(VCAD) [9], Modified Euclidean Distance[10] etc and those methods are discussed briefly below these methodologies do not focus on skin color matching.

\subsection{Manhattan Distance}

The Manhattan distance computes the sum of difference in each dimension of two vectors in $n$ dimensional vector space. It is the sum of the absolute differences of their corresponding components. Manhattan distance is also called the $L_{1}$ distance. If $u=\left(x_{1}, x_{2} \ldots x_{n}\right)$ and $v=\left(y_{1}, y_{2} \ldots . y_{n}\right)$ are two vectors in $\mathrm{n}$ dimensional hyper plane, then the Manhattan Distance $M D(u, v)$ between two vectors $\mathrm{u}, \mathrm{v}$ is given by the Eq. $\mathbf{1}$.

$$
\begin{aligned}
M D(u, v) & =\left|x_{1}-y_{1}\right|+\left|x_{2}-y_{2}\right|+\ldots .+\left|x_{n}-y_{n}\right| \\
= & \sum_{i=1}^{n}\left|x_{i}-y_{i}\right|
\end{aligned}
$$

Now for two RGB scale images of size $p \times q, I_{1}(a, b, c)$ and $I_{2}(a, b, c)$ where $a=1,2 \ldots . p$, $b=1,2 . . q$ and $c=1,2,3$ where c represents color intensity values Red, Green, Blue respectively. Manhattan Distance is measured using Eq. 2.

$$
M D\left(I_{1}, I_{2}\right)=\sum_{a=1}^{p} \sum_{b=1}^{q} \sum_{c=1}^{3}\left|I_{1}(a, b, c)-I_{2}(a, b, c)\right|
$$

As the number of pixels, $\mathrm{n}$ which falls in skin region varies with varying size of the image, so rather than taking the absolute distance further the distance is being normalized using Eq. 3 . 


$$
M D_{1}\left(I_{1}, I_{2}\right)=\frac{M D\left(I_{1}, I_{2}\right)}{n} ;
$$

where $n=$ number of pixels considered.

Manhattan distance between skin regions of the images shown in Fig 3(a) and Fig. 3(b) is 66.2244.

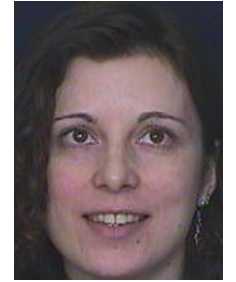

(a)

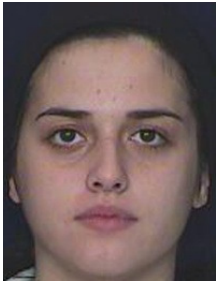

(b)

Figure 3. Manhattan distance between images=66.2244. (a) Facial image 1 (b)Facial Image 2.

\subsection{Euclidean Distance}

It is also called the $\mathrm{L}_{2}$ distance. For the same two vectors in $\mathrm{n}$ dimensional hyper plane, $u=\left(x_{1}, x_{2} \ldots x_{n}\right)$ and $v=\left(y_{1}, y_{2} \ldots . y_{n}\right)$, the Euclidean Distance $E D(u, v)$ is defined as Eq.4.

$$
\begin{aligned}
E D(u, v) & =\sqrt{\left(x_{1}-y_{1}\right)^{2}+\left(x_{2}-y_{2}\right)^{2}+. .+\left(x_{n}-y_{n}\right)^{2}} \\
& =\sqrt{\sum_{i=1}^{n}\left(x_{i}-y_{i}\right)^{2}}
\end{aligned}
$$

And for the same two RGB images $I_{1}(a, b, c), I_{2}(a, b, c)$, Euclidean Distance is measured using Eq. 5 .

$$
E D\left(I_{1}, I_{2}\right)=\sum_{a=1}^{p} \sum_{b=1}^{q} \sqrt{\sum_{c=1}^{3}\left(I_{1}(a, b, c)-I_{2}(a, b, c)\right)^{2}}
$$

Further the Euclidean distance is normalized using Eq. 6.

$$
E D_{1}\left(I_{1}, I_{2}\right)=\frac{E D_{1}\left(I_{1}, I_{2}\right)}{n}
$$

where $\mathrm{n}=$ number of pixels considered

Euclidean Distance between the skin regions of the same color facial images shown in Fig. $\mathbf{3}$ is 40.046 .

\subsection{Vector Cosine Angle Distance(VCAD)}

Vector Cosine Angle Distance metric [9] to measure distance between two points in $\mathrm{n}$ dimensional hyper plane which performs better where Euclidean Distance gives unsatisfactory results [9].Given two vectors $u=\left(x_{1}, x_{2} \ldots . x_{n}\right)$ and $v=\left(y_{1}, y_{2} \ldots . y_{n}\right)$ in a $\mathrm{n}$ dimensional hyper plane, then Vector Cosine Angle Distance, $\operatorname{VCAD}(u, v)$ is given by Eq. 7. 


$$
\operatorname{VCAD}(u, v)=\frac{\sum_{i} x_{i} y_{i}}{\sqrt{\sum_{i} x_{i}^{2}} \sqrt{\sum_{i} y_{i}^{2}}}=\frac{u . v}{\|u\|\|v\|}
$$

In the Fig. $4 \mathrm{VCAD}$ is shown for two vectors $u_{1}=\left(x_{1}, x_{2}\right)$ and $v_{1}=\left(y_{1}, y_{2}\right)$ in a two dimensional vector space.

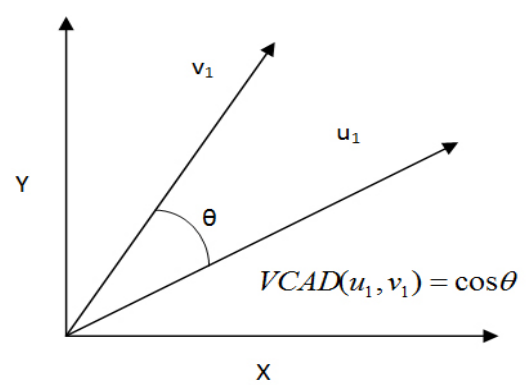

Figure 4. VCAD between vectors in two dimensional space.

From the figure it is clear that $V C A D \in[0,1]$. One important property of Vector cosine angle is that it gives a metric of similarity [9] between two vectors unlike Manhattan distance and Euclidean distance, both of which give metrics of dissimilarities [9]. For the same two RGB images of size $p \times q, I_{1}(a, b, c)$ and $I_{2}(a, b, c)$ then $\operatorname{VCAD}\left(I_{1}, I_{2}\right)$ is measured using Eq. 8 .

$$
\operatorname{VCAD}\left(I_{1}, I_{2}\right)=\frac{\sum_{a=1}^{p} \sum_{b=1}^{q} \sum_{c=1}^{3} I_{1}(a, b, c)^{*} I_{2}(a, b, c)}{\sqrt{\sum_{a=1}^{p} \sum_{b=1}^{q} \sum_{c=1}^{3} I_{1}(a, b, c)^{2}} \sqrt{\sum_{a=1}^{p} \sum_{b=1}^{q} \sum_{c=1}^{3} I_{2}(a, b, c)^{2}}}
$$

VCAD between the skin regions of the same color facial images shown in Fig. 3 is 0.9587.

\subsection{Modified Euclidean Distance proposed by Jain and Vailaya}

In RGB color space, color information in an image can be represented by a single 3D Histogram or three separate 1D histograms for each of the Red, Green, Blue .These histograms are invariant under rotation and translation of the input image. A suitable normalization of these histogram(s) also provides scale invariance. Let $H(i)$ be a histogram of an image, where index $i$ represents a histogram bin. Then the normalized histogram $I$ is defined as using Eq. 9.

$$
I(i)=\frac{H(i)}{\sum_{i} H(i)}
$$

Let $I_{R}, I_{G}$ and $I_{B}$ be the normalized color histograms of the colors Red, Green and Blue color channels of an RGB image $I . Q_{R}, Q_{G}$ and $Q_{B}$ be the normalized histograms of the colors Red, Green and Blue color channels of another image Q. Then Jain and Vailaya have redefined Euclidean distance [10] in a way that gives normalized metrics of similarity. They define the similarity between a query image and a stored database image $S_{c}{ }^{E D}(I, Q)$ is defined in Eq. 10 .

$$
S_{c}^{E D}=1.0-\frac{\sqrt{\sum_{r}\left(I_{R}(r)-Q_{R}(r)\right)^{2}+\sum_{g}\left(I_{G}(g)-Q_{G}(g)\right)^{2}+\sum_{b}\left(I_{B}(b)-Q_{B}(b)\right)^{2}}}{2 * 3}
$$


Note that the value of $S_{c}{ }^{E D}(I, Q) \in[0,1]$. If images $I$ and $Q$ are identical, then $S_{c}{ }^{E D}(I, Q)=1$.

Modified Euclidean distance using color histogram is applied on skin region of same color facial images shown in Fig. 3 is 0.9314.

\section{EXPERIMENTAL RESULTS}

The distance metrics discussed above has been applied on the images taken from standard FRAV2D database [11]. The present work has been implemented and tested on Matlab 7. Table I shows the distances calculated using the methodologies discussed of the image shown in Fig.5 to Fig. 9. Though full facial images have been shown in the Table I, all the methodologies discussed earlier have been applied on the facial images after manually removing the non-skin regions like hair, background using Adobe Photoshop CS2 version 9.0. Special care is taken while measuring the distance using the distance metrics on the regions where skin regions exist on both of the images. In other words only those pixels positions are considered which lie on the skin region of both of the images. First and second columns of the table shows the two facial images being considered, $3^{\text {rd }}$ and $4^{\text {th }}$ column shows the Manhattan and Euclidean distance respectively. Fifth and sixth column of Table I show Vector Cosine Angle Distance and Modified Euclidean Distance using color histogram respectively. In case of Manhattan and Euclidean distance less distance signifies more similarity but for VCAD and Modified Euclidean distance using color histogram higher values signifies more similarity.

Table I shows that the pair of images in figures $\{5(\mathrm{a}), 5(\mathrm{c})\},\{6(\mathrm{a}), 6(\mathrm{c})\},\{7(\mathrm{a}), 7(\mathrm{c})\},\{8(\mathrm{a})$, $8(\mathrm{c})\}$ and $\{9(\mathrm{a}), 9(\mathrm{c})\}$ are more similar in terms of complexion compared to the pair of images in figures $\{5(a), 5(b)\},\{6(a), 6(b)\},\{7(a), 7(b)\},\{8(a), 8(b)\}$ and $\{9(a), 9(b)\}$ respectively using the distances calculated by the methodologies of the Manhattan, Euclidean and Vector Cosine Angle. On the other hand, Modified Euclidean distance method concludes that the pairs of images in figures $\{5(\mathrm{a}), 5(\mathrm{c})\}$ and $\{7(\mathrm{a}), 7(\mathrm{c})\}$ are more similar in terms of complexion than the pair of images in figures $\{5(\mathrm{a}), 5(\mathrm{~b})\}$ and $\{7(\mathrm{a}), 7(\mathrm{~b})\}$ respectively. Only Modified Euclidean distance using color histogram shows that the pair of images in figures $\{6(a), 6(b)\},\{8(a), 8(b)\}$ and $\{9(a)$, 9 (b) $\}$ are more similar in terms of complexion compared to the pair of images $\{6(a), 6(c)\},\{8(a)$, $8(\mathrm{c})\}$ and $\{9(\mathrm{a}), 9(\mathrm{c})\}$ respectively.

Besides this, an opinion poll of 100 peoples has been taken to decide the similarity of two facial images in terms of complexion based on visual effect for all the facial images of FRAV2D database. The survey shows that the images of Fig. 5(a) to Fig. 8(a) is more similar in terms of complexion with Fig. 5(b) to Fig. 8(b) than the images of Fig. 5(c) to Fig.8(c) respectively which contradicts the results obtained from the Manhattan, Euclidean and Vector Cosine Angle Distance for all the images of Fig.5 to Fig.8. But results obtained from Modified Euclidean distance complies with the survey result for the images Fig. 6 and Fig. 8 only. The experimental result shows that result obtained by the methodologies of Manhattan, Euclidean and Vector Cosine Angle distance contradict the survey result in $80 \%$ cases and for Modified Euclidean distance methodology the contradiction arises in $60 \%$ cases. 


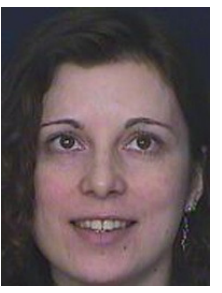

(a)

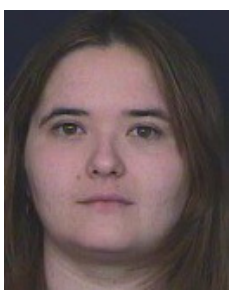

(b)

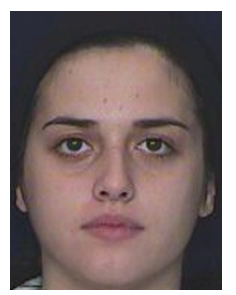

(c)

Figure 5. Three facial images taken from FRAV2D database to calculate the distance in terms complexion between them.(a)Sample image 1 (b) $1^{\text {st }}$ image for complexion comparison (c) $2^{\text {nd }}$ image for complexion comparison.

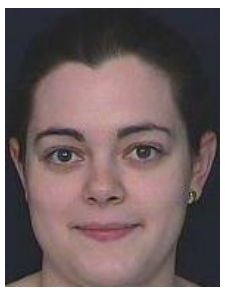

(a)

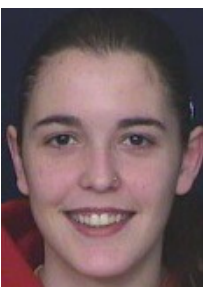

(b)

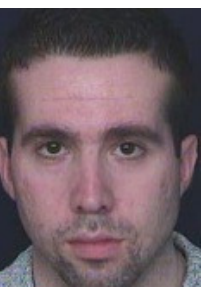

(c)

Figure 6. Three facial images taken from FRAV2D database to calculate the distance in terms complexion between them. (a)Sample image 2 (b) $1^{\text {st }}$ image for complexion comparison (c) $2^{\text {nd }}$ image for complexion comparison.

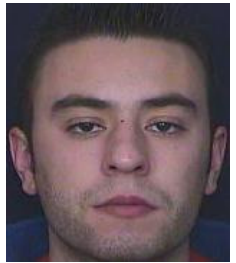

(a)

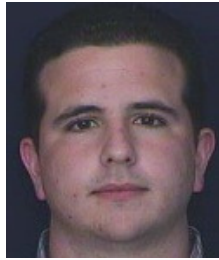

(b)

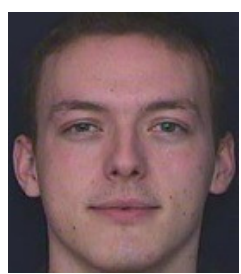

(c)

Figure 7. Three facial images taken from FRAV2D database to calculate the distance in terms complexion between them. (a)Sample image 3 (b) $1^{\text {st }}$ image for complexion comparison (c) $2^{\text {nd }}$ image for complexion comparison.

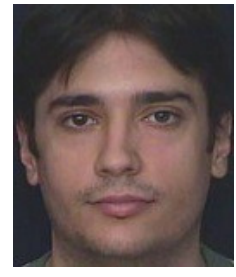

(a)

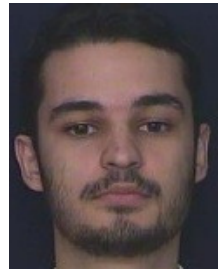

(b)

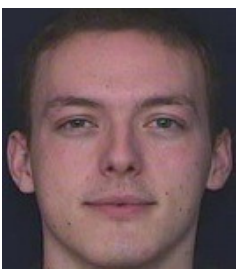

(c)

Figure 8. Three facial images taken from FRAV2D database to calculate the distance in terms complexion between them. (a)Sample image 2 (b) $1^{\text {st }}$ image for complexion comparison (c) $2^{\text {nd }}$ image for complexion comparison. 

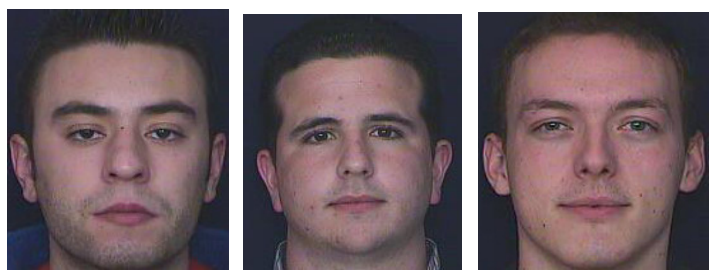

Figure 9. Three facial images taken from FRAV2D database to calculate the distance in terms complexion between them. (a)Sample image 2 (b)1st image for complexion comparison (c)2nd image for complexion comparison

TABLE I. ShOWS IMAGES AND THEIR RESPECTIVE MANHATTAN DistANCE

\begin{tabular}{|c|c|c|c|c|c|}
\hline \multirow{2}{*}{ Image 1 } & Image 2 & $\begin{array}{c}\text { Manhattan } * \\
\text { Distance }\end{array}$ & $\begin{array}{c}\text { Euclidean } \\
\text { Distance }\end{array}$ & VCAD** & $\begin{array}{c}\text { Modified } * * \\
\text { Euclidean }\end{array}$ \\
\hline \multirow{2}{*}{ Fig.5(a) } & Fig.5(b) & 148.3632 & 86.8941 & 0.8991 & 0.9215 \\
\cline { 2 - 6 } & Fig.5(c) & 66.2244 & 40.0460 & 0.9587 & 0.9314 \\
\hline \multirow{2}{*}{ Fig.6(a) } & Fig.6(b) & 90.7274 & 53.5984 & 0.9447 & 0.9216 \\
\cline { 2 - 6 } & Fig.6(c) & 80.5785 & 48.2347 & 0.9512 & 0.9172 \\
\hline \multirow{2}{*}{ Fig.7(a) } & Fig.7(b) & 119.4352 & 69.9836 & 0.9090 & 0.9312 \\
\cline { 2 - 6 } & Fig.7(c) & 82.2660 & 49.3977 & 0.9388 & 0.9525 \\
\hline \multirow{2}{*}{ Fig.8(a) } & Fig.8(b) & 129.3553 & 76.0788 & 0.8884 & 0.9373 \\
\cline { 2 - 6 } & Fig.8(c) & 84.9512 & 50.6457 & 0.9395 & 0.9113 \\
\hline \multirow{2}{*}{ Fig.9(a) } & Fig.9(b) & 87.2437 & 51.1189 & 0.9618 & 0.9659 \\
\cline { 2 - 6 } & Fig.9(c) & 69.2795 & 41.4078 & 0.9710 & 0.9361 \\
\hline
\end{tabular}

Fig. 10 and Fig. 11shows two snapshots of outputs taken using Matlab 7.

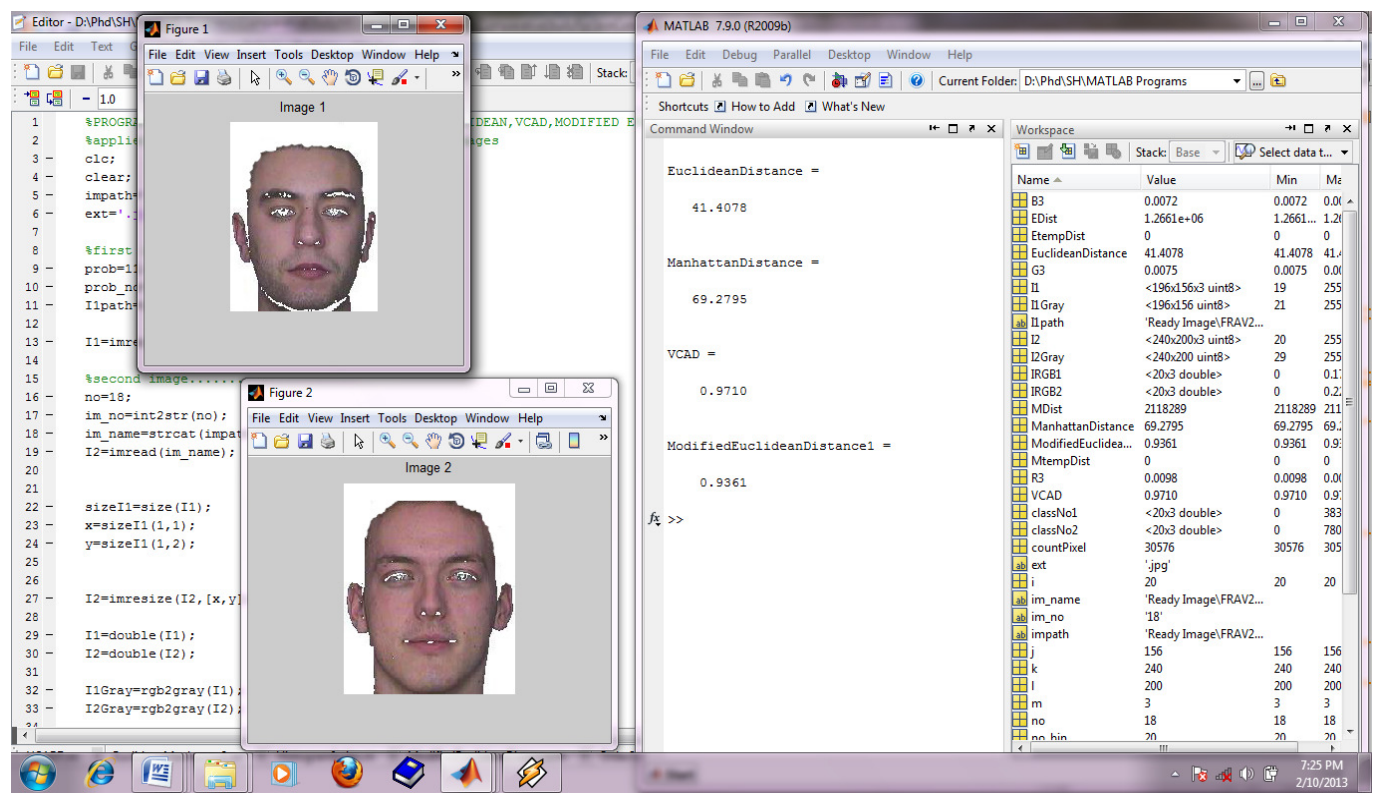


Figure 10. Snapshot of the output while measuring the distances between the pair of images in Fig. $\{9(\mathrm{a})$, $9(c)\}$.

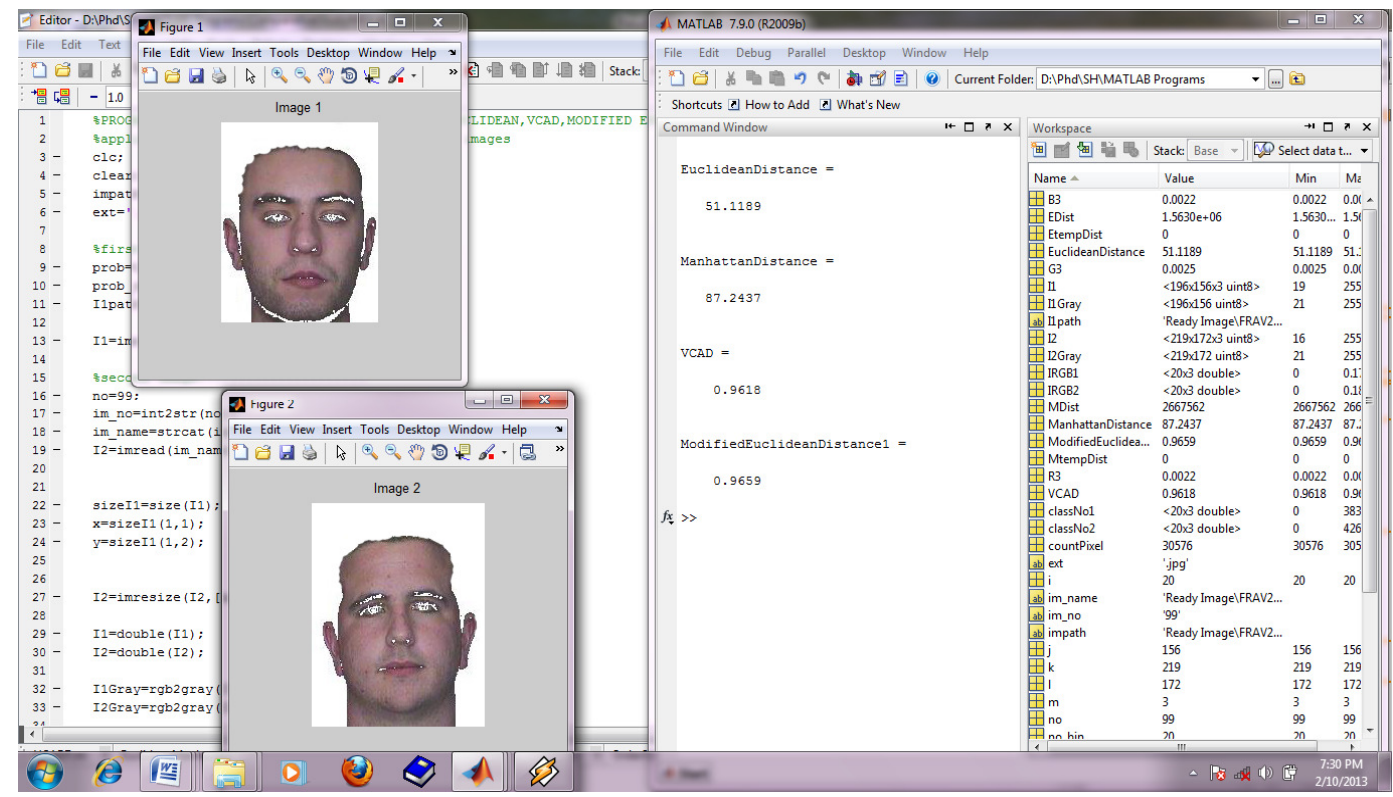

Figure 11. Snapshot of the output while measuring the distances between the pair of images in Fig. $\{9$ (a), $9(\mathrm{~b})\}$.

\section{CONCLUSiON}

This paper describes the comparative study of performance between the existing distance metrics like Manhattan, Euclidean, Vector Cosine Angle and Modified Euclidean distance for finding the similarity by calculating the distance between the skin colors of two facial images. The existing methodologies have been tested on 110 male and 40 female facial images taken from FRAV2D database. To verify the result obtained from the existing methodologies an opinion poll of 100 peoples have been taken. The experimental result shows that the result obtained by the methodologies of Manhattan, Euclidean and Vector Cosine Angle distance contradict the survey result in $80 \%$ cases and for Modified Euclidean distance methodology the contradiction arises in $60 \%$ cases.

\section{ACKNOWLEDGMENT}

Authors are thankful to the "Center for Microprocessor Application for Training Education and Research", "Project on Storage Retrieval and Understanding of Video for Multimedia" at Computer Science \& Engineering Department, Jadavpur University, for providing infrastructural facilities during progress of the work. Two of the authors, Dr. Santanu Halder and Mr. Abul Hasnat, are thankful to Government College of Engineering and Textile Technology, Berhampore, West Bengal for kindly permitting them to carry on the research work. 


\section{REFERENCES}

[1] Q.H. Thu, M. Meguro, M. Kaneko, (2002), "Skin-color extraction in images with complex background and varying illumination", Sixth IEEE Workshop on Applications of Computer Vision.

[2] D. Saxe, R. Foulds, (1996), "Toward robust skin identification in video images", AFGR96.

[3] S. McKenna, S. Gong, Y. Raja, (1998), "Modeling facial colour and identity with Gaussian mixtures", Pattern Recognition 31 (12) pp. 1883-1892.

[4] C. Garcia, G. Tziritas, (1999), "Face detection using quantized skin color regions merging and wavelet packet analysis", IEEE Trans. Multimedia 1 (3) pp. 264-277.

[5] N. Sebe, T. Cohen, T.S. Huang, T. Gevers,(2004), "Skin detection a Bayesian network approach", ICPR04.

[6] A. Hadid, M. Pietikinen and B. Martinkauppi, ( 2002), "Color-Based Face Detection using Skin Locus Model and Hierarchical Filtering”, Proceedings. 16th International Conference on Pattern Recognition, pp. $196-200$.

[7] D. Bhattacharjee, S. Halder , M. Nasipuri, D.K. Basu, M. Kundu, ( 2009), "Construction of Human Faces from Textual Descriptions", Soft Computing - A Fusion of Foundations, Methodologies and Applications, Vol. -15, No. - 3, pp. 429-447.

[8] D. Chai, and K.N. Nghan,(1999), "Face Segmentation using Skin Color Map in Videophone Applications", IEEE Transactions on Circuits and Systems for Video Technology, Vol. 9, No. 4.

[9] A Vadivel, A K Majumdar, Shamik Sural, ( 2003), "Performance comparison of distance metrics in content-based Image retrieval applications", International Conference on Information Technology (CIT), Bhubaneswar, India, pp. 159-164.

[10] Jain Anil K,Vailaya Aditya, (1996), "Image retrieval using color and shape", Pattern Recognition, Volume 29, Issue 8, Pages 1233-1244.

[11] FRAV2D Database (2004), Freely available from: http://www.frav.es/databases/frav2d/. 